\title{
Expression of Class III Beta-tubulin Predicts Prognosis in Patients with Cisplatin-resistant Bladder Cancer Receiving Paclitaxel-based Second-line Chemotherapy
}

\author{
YASUYOSHI MIYATA, TOMOHIRO MATSUO, YUICHIRO NAKAMURA, \\ TAKUJI YASUDA, KOJIRO OHBA, KOSUKE TAKEHARA and HIDEKI SAKAI
}

Department of Urology, Nagasaki University Graduate School of Biomedical Sciences, Nagasaki, Japan

\begin{abstract}
Background/Aim: Class III beta-tubulin (TUBB3) expression is recognized as a predictive marker for chemosensitivity to cisplatin- and taxane-based chemotherapies in various malignancies. The aim of this study was to clarify the predictive value of TUBB3 expression for the anticancer effects of first-line cisplatinbased chemotherapy and second-line paclitaxel-based chemotherapy in patients with urothelial cancer (UC). Patients and Methods: We reviewed 116 patients with UC (90 with bladder cancer and 27 with upper urinary tract cancer) treated with first-line cisplatin-based chemotherapy. Among them, 42 patients received a paclitaxel-based regimen as second-line chemotherapy for advanced cisplatin-resistant UC. TUBB3 expression was evaluated using immunohistochemistry, and survival analyses were performed using Kaplan-Meier survival curves and multivariate Cox proportional hazard analysis. Results: TUBB3 was mainly detected in the cytoplasm of cancer cells, and 64 patients (55.2\%) were judged as having positive TUBB3 expression. TUBB3 expression was significantly associated with tumour grade $(p<0.001)$. TUBB3 expression was not associated with time to progression after first-line cisplatin-based chemotherapy. However, positive expression of TUBB3 was significantly associated with unfavourable overall survival in patients receiving second-line paclitaxel-based chemotherapy $(p=0.021)$. In addition, a multivariate analysis model
\end{abstract}

This article is freely accessible online.

Correspondence to: Yasuyoshi Miyata, Department of Urology, Nagasaki University Graduate School of Biomedical Sciences, 1-71 Sakamoto, Nagasaki 852-8501, Japan. Tel: +81 958197340, Fax: +81 958197343, e-mail: int.doc.miya@m3.dion.ne.jp

Key Words: Class III beta-tubulin, cisplatin, paclitaxel, bladder cancer, prognosis. including T-stage and metastasis at the beginning of secondline therapy and regimen showed that TUBB3 expression was an independent predictor of poorer survival (hazard ratio $(H R)=3.44,95 \%$ confidential interval $(C I)=1.15-10.33$, $p=0.027)$. Conclusion: TUBB3 expression was identified as a useful predictive factor for survival after second-line paclitaxel-based therapy in patients with cisplatin-resistant UC. Our results are useful for determining treatment strategies for such patients

Bladder cancer (BC) is one of the most common malignancies, especially in industrialized countries. At initial diagnosis, $75 \%$ of patients have non-muscle-invasive bladder cancer, and the remaining $25 \%$ have muscle-invasive bladder cancer (1). Unfortunately, nearly one-third of patients with non-muscle invasion subsequently have regional invasion or metastasis (2). Although upper tract urothelial cancer (UC) is relatively rare, the prognosis is poor. In fact, the 5-year survival rate of patients with pT4 or metastasis of upper tract $\mathrm{UC}$ is only $10 \%$ (3). For such patients with advanced UC, chemotherapy is the most common and effective method to improve patient outcome. Therefore, information on predictive factors for the anticancer effects of chemotherapy in patients with UC is important for planning their treatment strategy.

Microtubules are cytoskeletal proteins built of $\alpha$ - and $\beta$ tubulin heterodimers (4). They are well-studied regulators of various cellular mechanisms, including maintenance of cell shape, intracellular transport, and cell mobility during mitosis and meiosis (5). Class III $\beta$-tubulin (TUBB3) is a $\beta$ tubulin isotype that has been reported to play important roles in malignant behaviour and prognosis of various types of malignancies $(6,7)$. Furthermore, many investigators have paid attention to the relationships between TUBB3 expression and efficacy of a variety of chemotherapeutic agents in patients with advanced stage malignancies. For example, in vivo and in vitro studies demonstrated that TUBB3 expression was associated with chemoresistance to 
taxanes in ovarian, breast, and lung cancer (8-10). In addition to its relationship with taxanes, several investigators reported that TUBB3 affected the response to DNAdamaging agents, including platinum-based agents, in lung cancer $(11,12)$. These reports support the opinion that TUBB3 overexpression is associated with poor outcome in patients treated with taxanes or platinum-based agents. However, other studies have shown that high expression of TUBB3 predicted better response to taxane-based chemotherapy in several types of cancer $(13,14)$. Likewise, there is a report that TUBB3 expression was not associated with chemosensitivity to cisplatin in non-small cell lung cancer (15). Thus, the predictive value of TUBB3 expression for chemosensitivity is not fully understood.

A recent report showed that TUBB3 overexpression is positively associated with malignant aggressiveness in $\mathrm{BC}$, via increased genetic instability, p53 alterations, and rapid cell proliferation (16). In addition, another report also showed that TUBB3 overexpression in BC cells was a significant predictor of recurrence-free survival in patients treated with radical cystectomy (17). Thus, relationships between TUBB3 expression and malignant aggressiveness, pathological features, and prognosis in patients with $\mathrm{BC}$ have already been investigated. However, the predictive value of TUBB3 expression for sensitivity to chemotherapeutic agents in patients with UC is not fully understood, despite platinum- or paclitaxel-based chemotherapy being commonly used to treat UC. A cisplatin-based regimen is recognized as the standard first-line chemotherapy for patients with locally invasive/metastatic UC. Although no second-line regimen for cisplatin-resistant UC hast yet been established, several clinical studies have demonstrated that paclitaxel-based chemotherapy is useful for improving the quality of life and prognosis in patients with cisplatin-resistant UC (18-20). In recent years, the efficacy of immune-checkpoint inhibitors was reported in patients with advanced $\operatorname{UC}(21,22)$. There is general agreement that this new tool is an epoch-changing therapeutic strategy for these patients. However, immunecheckpoint inhibitors are currently extremely expensive and have some severe side-effects. Therefore, we believe that effort should be focused on identifying those patients who are most likely to benefit from cisplatin- and paclitaxel-based regimens.

Based on these facts, the main aim of this study was to clarify the prognostic roles of TUBB3 expression in the efficacy of a cisplatin- and paclitaxel-based regimen in patients with advanced UC. In this study, the relationship between TUBB3 expression and time to progression (TTP) after first-line cisplatin-based chemotherapy was first investigated. Next, we analyzed the predictive value of TUBB3 expression for overall survival (OS) after second-line paclitaxel-based chemotherapy in patients with cisplatinresistant UC.
Table I. Patient characteristics and class III beta-tubulin (TUBB3) expression at the beginning of first-line chemotherapy.

\begin{tabular}{lcccc}
\hline Characteristic & $\begin{array}{c}\text { Number of } \\
\text { patients }(\%)\end{array}$ & $\begin{array}{c}\text { TUBB3 expression, } \\
\text { n }(\%)\end{array}$ & \\
\cline { 3 - 4 } & & $\begin{array}{c}\text { Negative } \\
(\mathrm{N}=52,\end{array}$ & $\begin{array}{c}\text { Positive } \\
(\mathrm{N}=64,\end{array}$ & \\
& & $44.8 \%)$ & $55.2 \%)$ & \\
& & & & $<0.001$ \\
Grade & & $5(100.0)$ & $0(0.0)$ & \\
1 & $5(43.1)$ & $53(62.2)$ & $14(37.8)$ & \\
2 & $37(31.9)$ & $23(32.4)$ & $50(67.6)$ & \\
3 & $74(63.8)$ & $24(3)$ & 0.062 \\
T-Stage & & & & \\
Ta+1 & $18(15.5)$ & $5(27.8)$ & $13(72.2)$ & \\
T2 & $45(38.8)$ & $29(64.4)$ & $16(35.6)$ & \\
T3 & $42(36.2)$ & $23(54.8)$ & $19(45.2)$ & \\
T4 & $11(9.5)$ & $7(63.6)$ & $4(36.4)$ & \\
Metastasis & & & & 0.727 \\
Absence & $24(20.7)$ & $10(41.7))$ & $14(58.3)$ & \\
Presence & $92(79.3)$ & $42(45.7)$ & $50(54.3)$ & \\
Chemtherapy regimen & & & & 0.822 \\
MVEC & 32 & 13 & 19 & \\
GC & 31 & 15 & 16 & \\
Intra-arterial & 53 & 24 & 29 & \\
\hline
\end{tabular}

MVEC: Methotrexate, vinblastine, epirubicin and cisplatin; GC: combination of gemcitabine and cisplatin.

\section{Patients and Methods}

Patients. A total of 116 patients with UC (31 men and 85 women; $90 \mathrm{BC}$ and 26 upper tract UC ) who were treated with chemotherapy at the Nagasaki University Hospital between 2003 and 2013 were analysed retrospectively. The median age at the beginning of firstline chemotherapy administration was 66 years (interquartile range $=60-75$ years). Clinicopathological features at the beginning of first-line chemotherapy are shown in Table I. Tumour grade was judged by histopathological examination in biopsy or transurethral resection specimens. T-Stage was diagnosed by imaging analysis, including enhanced computed tomography (CT) or magnetic resonance imaging (MRI). In this study, 18 patients with nonmuscle invasive disease were treated with cisplatin-based chemotherapy because the tumour size was large (over $5 \mathrm{~cm}$ ), the number of tumours was extremely high (over 20), or the patients were unable to undergo radical surgery as they were elderly or had metastatic disease. Metastasis was evaluated with chest radiography, abdominal and pelvic CT, pelvic MRI, and bone scintigraphy. Patients with pure squamous cell carcinoma or adenocarcinoma were excluded. This study protocol and the use of all materials were approved by the Institutional Review Board of Nagasaki University Hospital and written informed consent was obtained.

Treatment protocols. A schema of treatment progress is shown in Figure 1. All patients received cisplatin-based chemotherapy as a first-line regimen, and 42 patients underwent paclitaxel-based second-line chemotherapy for advanced UC. Another 11 patients were treated with gemcitabine monotherapy $(n=4)$ or best supportive 


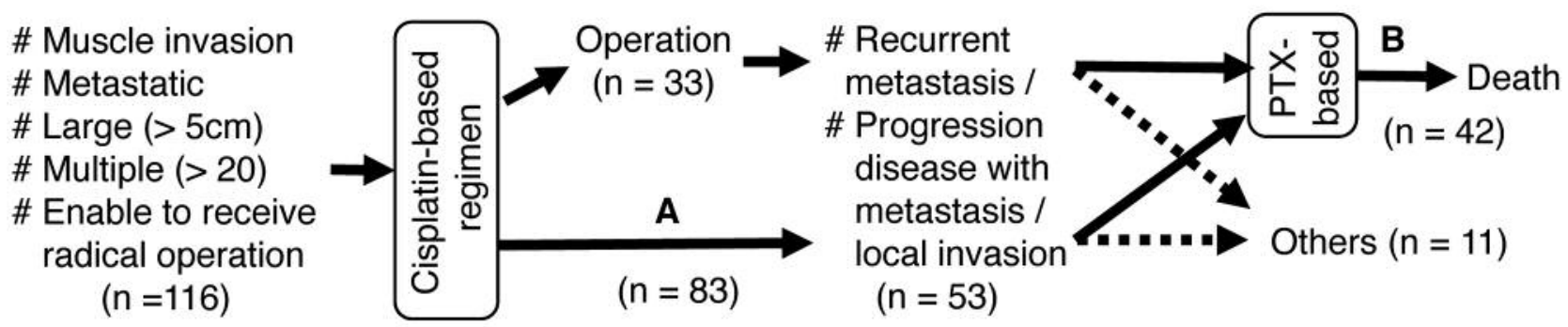

Figure 1. Schema of treatment progress of patients included in the study. Patients with urothelial cancer were treated with either first-line cisplatinbased chemotherapy alone (83 patients) (A) or after radical operation (33 patients). Patients who received chemotherapy alone were examined for time to progression. Patients who developed disease progression and received paclitaxel (PTX)-based chemotherapy as second-line treatment were examined for overall survival $(B)$.

care $(n=7)$. For cisplatin-based chemotherapy, methotrexate, vinblastine, epirubicin, and cisplatin (MVEC) regimen, gemcitabine and cisplatin (GC) regimen, and intra-arterial infusion of cisplatin were administered to $32(27.6 \%), 31(26.7 \%)$, and 53 patients $(45.7 \%)$, respectively. The MVEC regimen consisted of methotrexate at $30 \mathrm{mg} / \mathrm{m}^{2}$ on days 1,15 , and 22 ; vinblastine at 3 $\mathrm{mg} / \mathrm{m}^{2}$ on days 2,15 and 22 ; epirubicin at $30 \mathrm{mg} / \mathrm{m}^{2}$ on day 2 ; and cisplatin at $70 \mathrm{mg} / \mathrm{m}^{2}$ on day 2 , administered by intravenous infusion over a 28 -day cycle. GC therapy consisted of gemcitabine at $1,000 \mathrm{mg} / \mathrm{m}^{2}$ on days 1,8 , and 15 ; and cisplatin at $70 \mathrm{mg} / \mathrm{m}^{2}$ on day 2 , and was also administered by intravenous infusion over a 28day cycle. MVEC or GC regimens were continued until disease progression, unacceptable toxicity, or patient decision. In our study population, all patients receiving first-line MVEC or GC regimens received at least two cycles, and the median number of treatment cycles was four. In regard to intra-arterial therapy, cisplatin at 70 $\mathrm{mg} / \mathrm{m}^{2}$ and doxorubicin at $30 \mathrm{mg} / \mathrm{m}^{2}(1988-1994)$ or epirubicin at $30 \mathrm{mg} / \mathrm{m}^{2}$ (1993-present) were introduced in a 30- to $40-\mathrm{min}$ sequence. Two to four cycles of intra-arterial chemotherapy were administered (median=2).

For second-line therapy, paclitaxel plus gemcitabine therapy $(n=34)$ and paclitaxel plus carboplatin $(n=8)$ were administered. Gemcitabine at $700 \mathrm{mg} / \mathrm{m}^{2}$ and paclitaxel at $70 \mathrm{mg} / \mathrm{m}^{2}$ were administered by intravenous infusion on days 1 and 8 of each 28day cycle. Paclitaxel plus carboplatin therapy consisted of paclitaxel at $100 \mathrm{mg} / \mathrm{m}^{2}$ on days 1 and 8 , and carboplatin at an area under the curve of 5 on day 1 , administered by intravenous infusion over a 28-day cycle. Second-line treatment was continued until unacceptable toxicity or patient decision.

Immunohistochemistry. Five micrometre-thick sections were deparaffinized in xylene and rehydrated in ethanol. All sections were subjected to antigen retrieval and then immersed in hydrogen peroxide to block endogenous peroxidase activity. The antibody to TUBB3 was obtained from Abcam PLC (Cambridge, UK). Sections were incubated with the primary antibody at $4^{\circ} \mathrm{C}$ overnight. Then the sections were washed extensively and treated with peroxidase using the labelled polymer method with DAKO EnVision $+^{\mathrm{TM}}$ Peroxidase (Dako Corp., Carpinteria, CA, USA). The peroxidase reaction was visualized using the liquid 3,3'diaminobenzidine (DAB) substrate kit (Zymed Laboratories Inc., San Francisco, CA, USA). Sections were counterstained with haematoxylin.

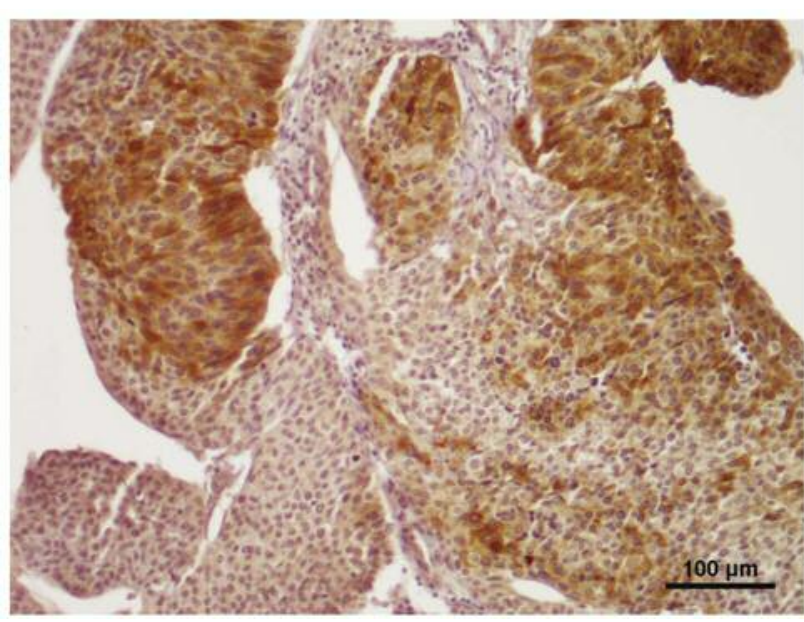

Figure 2. A representative example of class III beta-tubulin (TUBB3) expression in urothelial cancer tissue. TUBB3 expression was mainly detected in the cytoplasm of the cancer cells (original magnification $\times 200$ ).

Staining intensity was graded on a scale of 0 to 2 (no staining $=0$, weak $=1$, and strong $=2$ ). In addition, the extent of staining was scored as 0 : none, $1: 1-10 \%, 2: 11-50 \%$, or $3: 51-$ $100 \%$, according to the percentage of positively stained cancer cells. This proportional score was multiplied by the intensity score to obtain a semi-quantitative score, and specimens with scores of 4-6 were judged as having high expression of TUBB3, as described previously (15). Slides were examined using an E-400 microscope (Nikon, Tokyo, Japan) to produce digital images, which were examined using a computer-aided image analysis system (Win ROOF version 5.0; MITANI, Fukui, Japan). Slides were evaluated twice at different times by two investigators (YM and TM), who were blinded to the clinicopathological features and survival data.

Survival analyses. TTP and OS were calculated from the date therapy started to the date of disease progression and death, respectively. TTP after first-line cisplatin-based chemotherapy and OS after second-line paclitaxel-based chemotherapy were analysed 


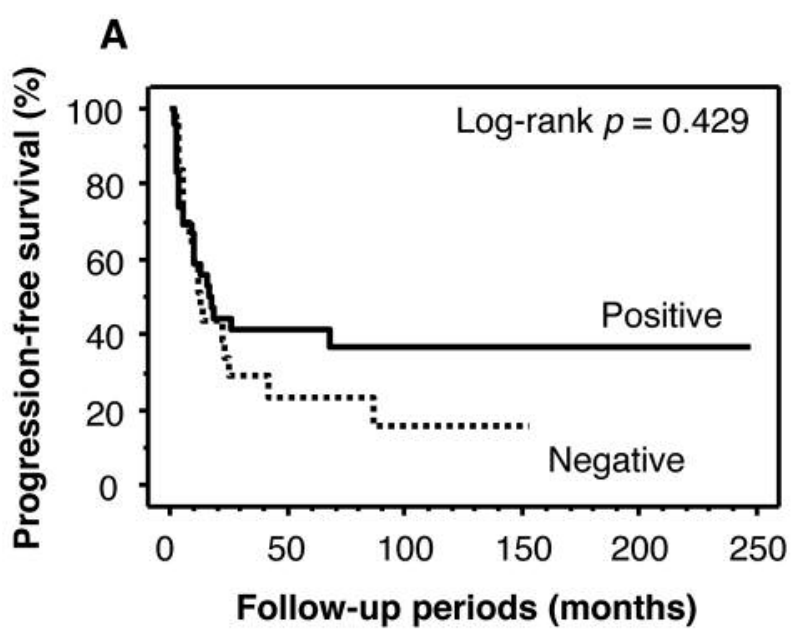

B

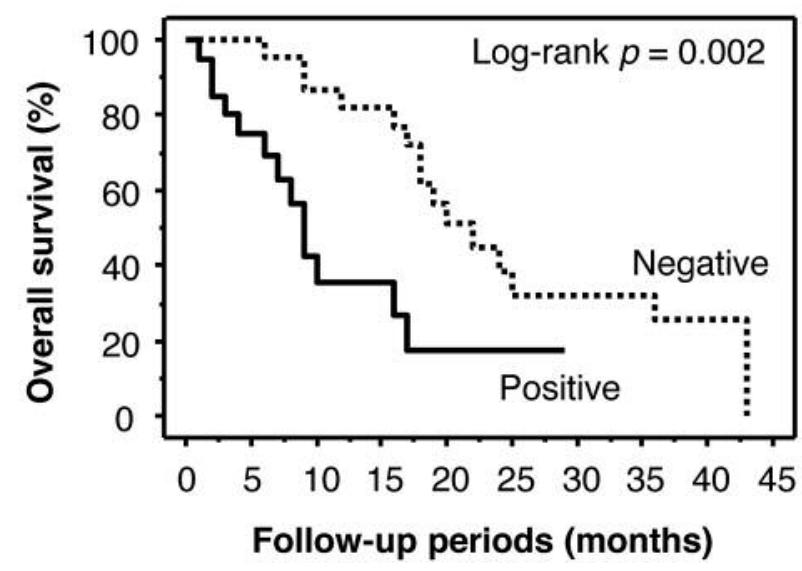

Figure 3. A: Kaplan-Meier survival curve showing time to progression in patients with urothelial cancer with high and low expression of class III beta-tubulin (TUBB3) after first-line cisplatin-based chemotherapy. B: Kaplan-Meier survival curve showing overall survival after second-line paclitaxel-based chemotherapy in patients with cisplatin-resistant urothelial cancer with high and low TUBB3 expression.

in 83 and 42 patients, respectively. Patients who were treated by radical operation were excluded for TTP analyses after therapy. OS after second-line paclitaxel-based therapy was analysed in patients with cisplatin-resistant UC.

Statistics. Student's t-test was used for comparisons involving continuous variables. The chi-square test was used to compare categorical data. Differences in survival were assessed using Kaplan-Meier curves, the log-rank test, and Cox regression analysis, and are expressed using hazard ratios (HRs), 95\% confidence intervals (95\% CIs), and $p$-values. All statistical analyses were two-sided and performed in StatView for Windows (version 5.0; Abacus Concepts, Inc., Berkeley, CA, USA). $p$-Values of less than 0.05 were considered representative of statistical significance.
Table II. Multivariate analysis of predictive values for overall survival after second-line paclitaxel-based therapy.

\begin{tabular}{lccc}
\hline Factor & HR & $95 \%$ CI & $p$-Value \\
\hline T-Stage at second-line therapy & & & \\
T3 & 0.77 & $0.25-2.36$ & 0.643 \\
T4 & 0.78 & $0.21-2.83$ & 0.701 \\
$\begin{array}{l}\text { Metastasis at second-line therapy } \\
\quad \text { Presence }\end{array}$ & 0.88 & $0.38-2.06$ & 0.767 \\
$\begin{array}{l}\text { Second-line regimen } \\
\quad \text { Paclitaxel+gemcitabine }\end{array}$ & 0.48 & $0.17-1.36$ & 0.166 \\
$\quad \begin{array}{l}\text { TUBB3 expression } \\
\quad \text { Positive }\end{array}$ & 3.44 & $1.15-10.33$ & 0.027 \\
\hline
\end{tabular}

TUBB3: Class III beta-tubulin; HR: hazard ratio; CI: confidence interval.

\section{Results}

TUBB3 expression and correlations with clinicopathological features. A representative example of TUBB3 expression is shown in Figure 2. TUBB3 was mainly found in the cytoplasm of cancer cells, and 64 cases (55.2\%) were judged as being positive for TUBB3 expression. TUBB3 positivity was similar between samples from BC and upper UC (49 patients $/ 54.4 \%$ and 15 patients $/ 57.7 \%$, respectively; $p=0.769)$.

Relationships between TUBB3 expression and grade, stage, and regimen are shown in Table I. The percentage of TUBB3-positive grade 3 tumors $(50 / 74=67.6 \%)$ was significantly higher $(p<0.001)$ than grade $1(0 / 5=0.0 \%)$ and grade $2(14 / 37=37.8 \%)$ tumors. A similar trend was found for T-stage; however, it did not reach significance $(p=0.062$; Table I). No significant relationship was found between TUBB3 level and metastasis $(p=0.727)$. There was no correlation between TUBB3 expression and first-line chemotherapy regimen.

Correlation with progression after cisplatin-based chemotherapy. As shown in Figure 3A, TUBB3 expression was not significantly associated with TTP after first-line cisplatin-based chemotherapy $(p=0.796)$. When a similar analysis was performed separately for patients with BC and upper tract $\mathrm{UC}$, no significant prognostic role was found for either ( $p=0.704$ and $p=0.816$, respectively). Similarly, the predictive value of TUBB3 expression was not influenced by first-line chemotherapy regimen (MVEC, $p=0.489$; GC, $p=0.852$; intra-arterial therapy, $p=0.229)$.

Correlation with survival after second-line paclitaxel-based therapy. In this study, 42 patients received second-line paclitaxel-based chemotherapy. At the beginning of second- 
line chemotherapy, light (19.0\%), 14 (33.3\%), and $20(47.6 \%)$ patients were diagnosed as having stage $\mathrm{T} 2, \mathrm{~T} 3$, and $\mathrm{T} 4$ tumour, respectively. Twenty-seven patients $(64.3 \%)$ had metastases. All patients were judged to have unresectable tumours. High expression of TUBB3 was significantly associated with unfavourable OS in patients receiving secondline paclitaxel-based chemotherapy ( $p=0.013$; Figure $3 \mathrm{~B}$ ). A multivariate analysis model including T-stage and metastatic status at the beginning of second-line chemotherapy and regimen of second-line therapy demonstrated that TUBB3 expression was independently associated with OS after second-line paclitaxel-based chemotherapy $(\mathrm{HR}=3.44$, 95\% CI=1.15-10.33, $p=0.027$; Table II).

\section{Discussion}

Our results demonstrated that high expression of TUBB3 was linked to poor outcome of second-line paclitaxel-based chemotherapy in patients with cisplatin-resistant UC. In addition, its expression was identified as an independent predictor in multivariate analysis models including tumour grade and stage. Unfortunately, as stated in the introduction, there are currently no established second-line chemotherapy regimens for cisplatin-resistant UC. However, there are several reports that paclitaxel-based chemotherapy has had some effectiveness in terms of improvement of quality of life and prognosis in patients with cisplatin-resistant UC $(18,23)$. In recent years, immune-checkpoint inhibitors have been reported as a novel treatment modality for patients with advanced UC, including platinum-treated advanced UC $(21,22)$. Many investigators and urologists have a great hope of increasing survival with these new agents in patients with advanced and cisplatinresistant UC; however, more careful evaluation of costeffectiveness, useful biomarkers, and safety are essential for standardization (21). Therefore, we believe that paclitaxelbased chemotherapy can immediately become a useful treatment option for patients with cisplatin-resistant UC. However, it is difficult to select patients most likely to benefit from paclitaxel-based chemotherapy. Thus, our finding that TUBB3 expression is a strong predictor for survival after second-line PTX-based chemotherapy is important information when discussing treatment strategies in cisplatin-resistant UC.

In contrast to second-line paclitaxel-based chemotherapy, our results showed that TUBB3 expression was not associated with TTP after first-line cisplatin-based chemotherapy. Cisplatin-based regimens are well-known as the most effective and standard method of first-line chemotherapy in patients with UC. On the other hand, cisplatin has relatively strong side-effects, such as nausea, bone marrow suppression, and alopecia. Therefore, prediction of the anticancer effects of cisplatin-based chemotherapy is important in planning treatment strategies. In several malignancies, increased expression of TUBB3 was reported to be significantly associated with poor prognosis and survival in patients receiving platinum-based chemotherapy $(24,25)$. Therefore, we expected that TUBB3 expression would be significantly associated with the anticancer effect of cisplatin-based chemotherapy in patients with advanced UC; however, our results did not support this hypothesis. Unfortunately, there is little information on the relationship between TUBB3 expression and the anticancer effects of cisplatin-based chemotherapy in patients with UC. On the other hand, some studies have shown that TUBB3 expression was not associated with prognosis in the adjuvant setting in non-small cell lung cancer (15). Ultimately, we suggest that the clinical prognostic role of TUBB3 expression for first-line cisplatin chemotherapy in patients with UC is minimal, and more detailed studies are necessary.

In this study, TUBB3 expression was significantly associated with tumor grade, but not T-stage or metastatic status. In various types of other malignancies, TUBB3 expression was positively associated with malignant aggressiveness, including grade, invasion, and metastasis (6, 7). Other investigators also reported that TUBB3 overexpression was associated with high grade in BC (17). We cannot explain the reason for the discrepancy between UC and other malignancies regarding the pathological significance of TUBB3. However, several other studies on a variety of malignancies have also repeated that TUBB3 expression is not associated with stage or metastatic status (7, 26-28). Finally, we speculate that methodological differences, such as antibodies used, method of evaluation, and background of patients, may contribute to this discrepancy, and that the pathological roles of TUBB3 in invasion and metastasis are minimal in UC. In addition, others have theorized that the malignant behaviour and pathological significance of TUBB3 is dependent on the cancer microenvironment $(6,29)$.

The major limitation of this study is that the sample size was relatively small. However, to our knowledge, this is the first study on the prognostic roles of TUBB3 for survival after second-line paclitaxel-based chemotherapy in patients with cisplatin-resistant UC. Another limitation is that the dose and schedule of the different regimens were different from patient to patient, according to tolerability and toxicity profile, because this was a retrospective analysis. However, no patients were treated with a remarkably different protocols because all treatments were performed at a single institution. We believe that these results are important for the discussion and determination of treatment strategies in patients with advanced UC, although more detailed studies are necessary to draw a firm conclusion on the predictive value of TUBB3 expression. 


\section{Conclusion}

Our results demonstrated that TUBB3 expression is a useful predictive factor for OS after second-line paclitaxel-based chemotherapy, but not for TTP after first-line cisplatin-based chemotherapy in patients with UC. Currently, paclitaxelbased second-line chemotherapy for cisplatin-resistant UC is a useful therapeutic tool. Therefore, our results are important for determining the optimal treatment strategies in these patients.

\section{Conflicts of Interest}

The Authors declare that they have no competing interests in regard to this study.

\section{References}

1 Messing EM, Young TB, Hunt VB, Gilchrist KM, Newton MA, Bram LL, Hisgen WJ, Greenberg EB, Kuglitsch ME and Wegenke JD: Comparison of bladder cancer outcome in men undergoing hematuria home screening versus those with standard clinical presentation. Urology 45: 387-396, 1995.

2 Sylvester RJ, van der Meijden APM, Oosterlinck W, Witjes JA, Bouffioux C, Denis L, Newling DW and Kurth K: Practing recurrence and progression in individual patients with stage $\mathrm{Ta}$ T1 bladder cancer using EORTC risk tables: a combined analysis of 2596 patients from seven EORTC trials. Eur Urol 49: 466477, 2006.

3 Hall MC, Womack S, Sagalowsky AI, Carmody T, Erickstad MD and Roehrborn CG: Prognostic factors, recurrence and survival in transitional cell carcinoma of the upper urinary tract: a 30year experience in 252 patients. Urology 52: 594-601, 1998.

4 Desai A, Mitchison, TJ: Microtubule polymerization dynamics. Annu. Rev. Cell Dev. Biol 13: 83-117, 1997.

5 Orr GA, Verdier-Pinard $\mathrm{P}$ and McDaid $\mathrm{H}$ and Horwitz SB: Mechanisms of taxol resistance related to microtubules. Oncogene 22: 7280-7295, 2003.

6 Mariani M, karki R, Spennato M, Pandya D, He S, Andreoli M, Fiedler P and Ferlini C: Class III $\beta$-tubulin in normal and cancer tissues. Gene 563: 109-114, 2015.

7 Lebok P, Öztürk M, Heilenkötter U, Jaenicke F, Müller V, Paluchowski P, Geist S, Wilke C, Burandt E, Lebeau A, Wilczak W, Krech T, Simon R, Sauter G and Quaas A: High levels of class III $\beta$-tubulin expression are associated with aggressive tumor features in breast cancer. Oncol Lett 11: 1987-1994, 2016.

8 Du J, Li B, Fang Y, Liu Y, Wang Y, Li J, Zhou W and Wang X: Overexpression of Class III $\beta$-tubulin, SOX2, and nuclear survivin is predictive of taxane resistance in patients with stage III ovarian epithelial cancer. BMC Cancer 15: 536, 2015.

9 Lopus M, Smiyun G, Miller H, Oroudjev E, Wilson L and Jordan MA: Mechanism of action of ixabepilone and its interactions with the $\beta$ III-tubulin isotype. Cancer Chemother Pharmacol 76: 1013-1024, 2015.

10 Ohashi T, Yoshimasu T, Oura S, Kokawa Y, Kawago M, Hirai Y, Miyasaka M, Aoishi Y, Kiyoi M, Nishiguchi H, Honda M and Okamura Y: Class III beta-tubulin expression in non-small cell lung cancer: a predictive factor for paclitaxel response. Anticancer Res 35: 2669-2674, 2015.
11 Gan PP, Pasquier E and Kavallaris M: Class III beta-tubulin mediates sensitivity to chemotherapeutic drugs in non small cell lung cancer . Cancer Res 67: 9356-9363, 2007.

12 Sun S, Shi W, Wu Z, Zhang G, Yang BO and Jiao S: Prognostic significance of the mRNA expression of ERCC1, RRM1, TUBB3 and TYMS genes in patients with non-small cell lung cancer. Exp Ther Med 10: 937-941, 2015.

13 Galmarini CM, Treilleux I, Cardoso F, Bernard-Marty C and Durbecq V, Gancberg D, Bissery MC, Paesmans M, Larsimont D, Piccart MJ, Di Leo A and Dumontet C: Class III beta-tubulin isotype predicts response in advanced breast cancer patients randomly treated either with single-agent doxorubicin or docetaxel. Clin Cancer Res 14: 4511-4516, 2008.

14 Aoki D, Oda Y, Hattori S, Taguchi K, Ohishi Y, Basaki Y, Oie S, Suzuki N, Kono S, Tsuneyoshi M, Ono M, Yanagawa T and Kuwano M: Overexpression of class III beta-tubulin predicts good response to taxane-based chemotherapy in ovarian clear cell adenocarcinoma. Clin Cancer Res 15: 1473-1480, 2009.

15 Huang ZL, Cao X, Luo RZ, Chen YF, Zhu LC and Wen Z: Analysis of ERCC1, BRCA1, RRM1 and TUBB3 as predictors of prognosis in patients with non-small cell lung cancer who received cisplatin-based adjuvant chemotherapy: A prospective study. Oncol Lett 11: 299-305, 2016.

16 Hinsch A, Chaker A, Burdelski C, Koop C, Tsourlakis MC, Steurer S, Rink M, Eichenauer TS, Wilczak W, Wittmer C, Fisch M, Simon R, Sauter G, Büschek F, Clauditz T, Minner S, Jacobsen F. $\beta$ III-Tubulin overexpression is linked to agressive tumor features and genetic instability in urinary bladder cancer. Hum Pathol 61: 210-220, 2017.

17 Choi JW, Kim Y, Lee JH and Kim YS: Expression of $\beta$-tubulin isoform in urothelial carcinoma of the bladder. World J Urol 32: 347-352, 2014.

18 Miyata Y, Nomata K, Ohba K, Matsuo T, Sagara Y, Kanetake $\mathrm{H}$ and Sakai $\mathrm{H}$ : Use of low-dose combined therapy with gemcitabine and paclitaxel for advanced urothelial cancer patients with resistance to cisplatin-containing therapy: a retrospective analysis. Cancer Chemother Pharmacol 70: 451459, 2012.

19 Miyata Y, Asai A, Mitsunari K, Matsuo T, Ohba K and Sakai H: Safety and efficacy of combination therapy with low-dose gemcitabine, paclitaxel, and sorafenib in patients with cisplatinresistant urothelial cancer. Med Oncol 32: 235, 2015.

20 Necchi A, Pond GR, Raggi D, Giannatempo P, Vogelzang NJ, Grivas P, Galsky MD, Bellmunt J and Sonpavde G: Efficacy and safety of gemcitabine plus either taxane or carboplatin in the first-line setting of metastatic urothelial carcinoma: a systematic review and meta-analysis. Clin Genitourin Cancer 15: 23-30.e2, 2017.

21 Tsiatas $\mathrm{M}$ and Grivas P: Immunobiology and immunotherapy in genitourinary malignancies. Ann Transl Med 4: 270, 2016.

22 Bellmunt J, Powles T and Vogelzang NJ: A review on the evolution of PD-1/PD-L1 immunotherapy for bladder cancer: The future is now. Cancer Treat Rev 54: 58-67, 2017.

23 Sideris S, Aoun F, Zanaty M, Martinez NC, Latifyan S, Awada A and Gil T: Efficacy of weekly paclitaxel treatment as a single agent chemotherapy following first-line cisplatin treatment in urothelial bladder cancer. Mol Clin Oncol 4: 1063-1067, 2016.

24 Koh Y, Kim TM, Jeon YK, Kwon TK, Hah JH, Lee SH, Kim DW, Wu HG, Rhee CS, Sung MW, Kim CW, Kim KH and Heo 
DS: Class III beta-tubulin, but not ERCC1, is a strong predictive and prognostic marker in locally advanced head and neck squamous cell carcinoma. Ann Oncol 20: 1414-1419, 2009.

25 Ting S, Mairinger FD, Hager T, Welter S, Eberhardt WE, Wohlschlaeger J, Schmid KW and Christoph DC: ERCC1, MLH1, MSH2, MSH6, and $\beta$ III-tubulin: resistance proteins associated with response and outcome to platinum-based chemotherapy in malignant pleural mesothelioma. Clin Lung Cancer 14: 558-567.e3, 2013.

26 Hwang JE, Hong JY, Kim K, Kim SH, Choi WY, Kim MJ, Jung SH, Shim HJ, Bae WK, Hwang EC, Lee KH, Lee JH, Cho SH and Chung IJ: Class III $\beta$-tubulin is a predictive marker for taxane-based chemotherapy in recurrent and metastatic gastric cancer. BMC Cancer 13: 431, 2013.

27 Powell S, Kaizer A, Koopmeiners JS, Iwamoto C and Klein M: High expression of class III $\beta$-tubulin in small cell lung carcinoma. Oncol Lett 7: 405-410, 2017.
28 Cao Y, Zhang G, Wang P, Zhou J, Gan W, Song Y, Huang L, Zhang Y, Luo G, Gong $\mathrm{J}$ and Zhang L: Clinical significance of UGT1A1 polymorphism and expression of ERCC1, BRCA1, TYMS, RRM1, TUBB3, STMN1 and TOP2A in gastric cancer. BMC Gastroenterol 17: 2, 2017.

29 Raspaglio G, Filippetti F, Prislei S, Raspaglio G, Filippetti F, Prislei S, Penci R, De Maria I, Cicchillitti L, Mozzetti S, Scambia G and Ferlini C: Hypoxia induces class III beta-tubulin gene expression by HIF-1alpha binding to its 3' flanking region. Gene 409: 100-108, 2008. 